\title{
Influencia de la variedad del castaño en la incidencia de insectos carpófagos en las principales zonas productoras de castañas en Andalucía
}

\author{
Cuestas, M.I. ${ }^{1}$, Martín, M.A. ${ }^{2}$, Martín, L.M. ${ }^{1}$, Aldebis, H.K. ${ }^{3}$, Mena, J.D. ${ }^{3}$, \\ Vargas Osuna, E. ${ }^{3 *}$
}

${ }^{1}$ Departamento de Genética, Universidad de Córdoba. Campus de Rabanales, Carretera Madrid Km. 396, 14014-Córdoba, España.

\begin{abstract}
${ }^{2}$ Departamento de Ingeniería del Medio Agronómico y Forestal, Universidad de Extremadura. ES-10600.
${ }^{3}$ Departamento de Ciencias y Recursos Agrícolas y Forestales, Universidad de Córdoba. Campus de Rabanales, Carretera Madrid Km. 396, 14014-Córdoba, España.
\end{abstract}

*Autor para correspondencia: cr1vaose@uco.es

\begin{abstract}
Resumen
Entre los fitófagos del castaño en Andalucía destacan insectos carpófagos cuyas larvas se desarrollan en el interior de las castañas ocasionando graves pérdidas de producción y depreciando comercialmente el fruto. El objetivo de este trabajo es conocer la influencia de la variedad en la intensidad de los daños en las dos principales zonas productoras de castaña en Andalucía: Valle del Genal (Málaga) y Sierra de Aracena (Huelva). Para ello se seleccionaron castaños de las principales variedades locales de cada zona, caracterizados genéticamente mediante marcadores microsatélites (SSRs), y se tomaron muestras aleatorias de 25 erizos por árbol para proceder al recuento de los daños por carpófagos. El daño principal correspondió casi exclusivamente a Cydia splendana, con diferencias significativas entre las dos zonas, siendo mucha menor la incidencia en Málaga que en Huelva. En la zona de Málaga la mayor incidencia correspondió a la variedad 'Temprana de Jubrique' que difirió significativamente respecto a 'Tomasa' y 'Pilonga'. En la zona de Huelva, las variedades 'Planta Alájar', 'Comisaria' y 'Helechal' tuvieron un mayor porcentaje de daños que la variedad 'Temprana'. No obstante, se pretende confirmar estos resultados en sucesivos años ampliando para cada variedad el número de castaños y fincas.
\end{abstract}

Palabras clave: caracterización varietal, Castanea sativa, Cydia splendana, daños, susceptibilidad. 


\section{Introducción}

Los castañares del Valle del Genal (Málaga) y de la Sierra de Aracena (Huelva) constituyen importantes recursos ecológicos, económicos y sociales que actualmente están gravemente amenazados por bajos rendimientos de producción debido principalmente a problemas fitosanitarios causados por plagas (Cobos, 1989; Mansilla et al., 2000), en especial por especies carpófagas entre las que destacan el lepidóptero Cydia splendana (Hb.) y el coleóptero Curculio elephas (Gyll.). Las pérdidas más importantes son ocasionadas por el tortrícido C. splendana, ampliamente distribuido por toda Europa, cuya presencia se extiende desde la Península Ibérica hasta el norte de Irán y Montes Urales. Las hembras depositan los huevos en las hojas cercanas a los erizos y las larvas neonatas se introducen en los frutos para alimentarse y desarrollarse en su interior. Cuando abandonan la castaña, ésta ha sufrido una disminución de peso y una pérdida de calidad por la presencia de excrementos y seda de la larva (Mansilla et al., 2000).

Los daños directos por carpófagos en Castanea sativa Mill. pueden llegar a causar reducciones de hasta el $60 \%$ en la producción de castaña, con porcentajes medios de daños en Andalucía relativamente altos aunque muy variables, entre 5 y $40 \%$ de las castañas según zona y finca (Romero, 2013). Esta variabilidad puede ser debida, entre otros factores, a diferencias de susceptibilidad entre variedades; sin embargo, el factor variedad no ha sido estudiado hasta la fecha, puesto que es necesaria la caracterización previa de las variedades locales a partir de las numerosas denominaciones varietales de uso común en cada zona.

Los marcadores moleculares denominados microsatélites SSRs (Simple Sequence Repeats) son los marcadores más empleados en la actualidad en estudios de diversidad genética debido a su alta reproductividad, naturaleza multialélica, forma de herencia codominante y amplia cobertura del genoma (Schlötterer, 2004). Trabajos realizados en especies vegetales con dichos marcadores han aportado una cuantiosa información sobre diversidad genética en poblaciones de especies forestales como nogal (Vahdati et al., 2015), pino (Fady, 2012), roble (Steinkellner et al., 1997) y castaño (Martín et al., 2010; Mattioni et al., 2013; Lusini et al., 2014). Además, se han convertido en las mejores herramientas para los estudios de identificación varietal en especies vegetales o de huella genética (Varshney et al., 2005). En castaño, la identificación de las variedades se está empezando a clarificar mediante el uso de estos marcadores (Gobbin et al., 2007; Martín et al., 2010; Pereira-Lorenzo et al., 2010). Concretamente, su uso ha mostrado la existencia de una amplia diversidad genética y ha permitido identificar variedades locales de castaño propias de Huelva y de Málaga, sin que existieran variedades comunes a ambas zonas (Martín et al., 2009).

Los objetivos de este estudio han sido:

1. Ampliar el número de individuos de cada variedad caracterizándolos genéticamente mediante marcadores SSRs.

2. Conocer si existen diferencias intervarietales en la incidencia de carpófagos en las principales zonas productoras de fruto en Andalucía. 


\section{Material y métodos}

\subsection{Material vegetal}

Un total de 24 árboles de variedades locales de las zonas del Valle del Genal (Málaga) y de la Sierra de Aracena (Huelva) fueron seleccionados de distintas fincas de cada zona para el estudio, concretamente de las variedades: 'Pilonga', 'Temprana de Jubrique' y 'Tomasa' en los términos municipales de Jubrique y Benadalid (Málaga) y las variedades 'Comisaria', 'Helechal', 'Planta Alájar' y 'Temprana 2' en Fuenteheridos, Cortegana y Los Marines (Huelva). La selección incluyó árboles que habían sido identificados en trabajos anteriores mediante marcadores moleculares SSRs (Martín et al., 2009) y nuevos árboles, para ampliar el número de individuos, de los que se tomaron muestras de hojas para su posterior análisis molecular e identificación varietal.

\subsection{Extracción de ADN y amplificación con SSRS}

El ADN fue extraído a partir de $20 \mathrm{mg}$ de hoja liofilizada siguiendo el protocolo de Murray et al. (1980) con modificaciones (ajustando las concentraciones del buffer de extracción). La identificación varietal se hizo utilizando un set de 8 microsatélites polimórficos desarrollados en C. sativa (Buck et al., 2003; Marinoni et al., 2003) (Tab. 1). La mezcla de reacción se hizo usando el kit Type-it Microsatellite PCR (QIAGEN, Hilden, Germany) en $12.5 \mu 1$ de volumen total con una concentración de cebadores de $2 \mu \mathrm{M}$ y $12 \mathrm{ng}$ de $\mathrm{ADN}$ genómico, según las indicaciones del protocolo. Los productos de amplificación fueron visualizados en gel de agarosa al 2\% para comprobar la fiabilidad de la amplificación. El análisis de fragmentos se llevó a cabo por parte de la Unidad de Genómica del Servicio Central de Apoyo a la Investigación (SCAI). El análisis de los datos se realizó utilizando el software Genotyper 3.7 (Applied Biosystems).

Tabla 1. Motivos de repetición y longitud del producto de cada SSR usados para la caracterización de las variedades.

\begin{tabular}{lll}
\hline Nombre del SSR & Motivo de repetición & Tamaño (pb) \\
\hline CsCAT1 & $(\mathrm{TG})_{25}$ TA $(\mathrm{TG})_{24}$ & $175-221$ \\
CsCAT2 & $(\mathrm{AG})_{16}$ & $193-233$ \\
CsCAT3 & $(\mathrm{AG})_{20}$ & $197-257$ \\
CsCAT6 & $(\mathrm{AC})_{24} \mathrm{AT}(\mathrm{AC})_{4}$ & $156-195$ \\
CsCAT14 & $(\mathrm{CA})_{22}$ & $130-164$ \\
CsCAT16 & $(\mathrm{TC})_{20}$ & $124-145$ \\
EMCs25 & $(\mathrm{GA})_{12}$ & $141-192$ \\
EMCs38 & $(\mathrm{AG})_{31}$ & $233-276$ \\
\hline
\end{tabular}




\subsection{Incidencia de carpófagos en los frutos}

Para determinar los daños en frutos se tomaron, al final de la campaña de 2014, muestras aleatorias de 25 erizos por árbol, que fueron llevados al laboratorio para proceder al recuento de castañas dañadas y determinar las especies carpófagas mediante las características morfológica de las larvas. Posteriormente, cada larva fue mantenida en incubadora a $15^{\circ} \mathrm{C}$ dentro de una caja de plástico, con rejilla de ventilación, con un trozo de castaña $\left(1 \mathrm{~cm}^{2}\right.$ aprox.) para su desarrollo hasta adulto.

\subsection{Análisis estadísticos}

Teniendo en cuenta que se trata de variedades diferentes en cada zona, se ha evaluado la incidencia de carpófagos asumiendo que la distribución de los árboles se puede asimilar a un diseño completamente aleatorizado para cada zona independientemente. La diferencia entre las dos zonas de estudio y el efecto de la variedad en los niveles de daño se analizaron mediante ANOVA, una vez que se comprobó la homogeneidad de varianzas y que los datos se ajustaban a una distribución normal. En los casos en que el ANOVA fue significativo, las medias entre variedades se compararon mediante el test LSD ( $\operatorname{con} \alpha=0.05$ ). El programa utilizado fue Statistix 9.0.

\section{Resultados}

El uso de los marcadores moleculares ha permitido la caracterización varietal de nuevos individuos ampliando así el número de árboles para conseguir un mínimo de 3 árboles por variedad.

El porcentaje de incidencia de $C$. splendana varió entre 0 y 48.6 en los árboles de la zona de Málaga, mientras que en Huelva este valor osciló entre 17.3 y 71.4 (Tab. $2)$, resultando ser altamente significativas las diferencias entre ambas zonas $(p<0.0001)$ (Tab. 2; Fig. 1). En algunos de los frutos recogidos se observaron también larvas de C. elephas, que en las muestras de Málaga aparecieron asociadas a un solo castaño, concretamente de la variedad 'Tomasa' con un 39.7\% de frutos infestados, mientras que en Huelva este fitófago afectó a 4 árboles de las variedades 'Comisaria', 'Helechal' y 'Planta Alájar', aunque en ninguno de los casos el grado de infestación superó el 4\% (Tab. 2).

En la zona de Málaga se encontraron diferencias significativas entre variedades $(p<0.05)$. La variedad 'Temprana de Jubrique' fue la que mostró niveles de daño más elevados con más del $26 \%$ de frutos infestados, mientras que la incidencia en 'Pilonga' y 'Tomasa' fue muy baja. Las diferencias intervarietales en el caso de Huelva no fueron tan marcadas, aunque también significativas $(p<0.05)$, siendo las variedades 'Planta Alájar', 'Comisaria' y 'Helechal' las que presentaron mayores niveles de daño (entre 40 y 50\%), mientras que en la variedad 'Temprana' no llegó al 20\% (Fig. 2). 
Tabla 2. Niveles de incidencia de Cydia splendana y Curculio elephas (en porcentaje de frutos dañados) según zona, variedad y árbol.

\begin{tabular}{|c|c|c|c|c|}
\hline Zona & Variedad & Ref. árbol & C. splendana & C. elephas \\
\hline Málaga & $\begin{array}{l}\text { Pilonga } \\
\text { Pilonga } \\
\text { Pilonga } \\
\text { Pilonga } \\
\text { Temprana de Jubrique } \\
\text { Temprana de Jubrique } \\
\text { Temprana de Jubrique } \\
\text { Tomasa } \\
\text { Tomasa } \\
\text { Tomasa } \\
\text { Tomasa }\end{array}$ & $\begin{array}{l}\text { MA-110 } \\
\text { MA-101 } \\
\text { MA-104 } \\
\text { MA-37 } \\
\text { MA-111 } \\
\text { MA-112 } \\
\text { MA-09 } \\
\text { MA-95 } \\
\text { MA-97 } \\
\text { MA-61 } \\
\text { MA-62 }\end{array}$ & $\begin{array}{c}0 \\
1.5 \\
5.0 \\
9.6 \\
18.7 \\
48.6 \\
12.1 \\
1.6 \\
3.2 \\
1.7 \\
14.3\end{array}$ & $\begin{array}{c}0 \\
0 \\
0 \\
0 \\
0 \\
0 \\
0 \\
0 \\
0 \\
0 \\
39.4\end{array}$ \\
\hline Huelva & $\begin{array}{l}\text { Comisaria } \\
\text { Comisaria } \\
\text { Comisaria } \\
\text { Helechal } \\
\text { Helechal } \\
\text { Helechal } \\
\text { Planta Alájar } \\
\text { Planta Alájar } \\
\text { Planta Alájar } \\
\text { Planta Alájar } \\
\text { Temprana } 2 \\
\text { Temprana } 2 \\
\text { Temprana } 2\end{array}$ & $\begin{array}{l}\text { HU-60 } \\
\text { HU-64 } \\
\text { HU-80 } \\
\text { HU-79 } \\
\text { HU-82 } \\
\text { HU-65 } \\
\text { HU-59 } \\
\text { HU-66 } \\
\text { HU-81 } \\
\text { HU-25 } \\
\text { HU-32 } \\
\text { HU-34 } \\
\text { HU-33 }\end{array}$ & $\begin{array}{l}51.2 \\
37.3 \\
57.3 \\
53.3 \\
41.3 \\
33.3 \\
40.0 \\
41.7 \\
50.7 \\
71.4 \\
23.5 \\
18.7 \\
17.3\end{array}$ & $\begin{array}{c}0 \\
4.0 \\
0 \\
0 \\
4.0 \\
1.3 \\
0 \\
0 \\
2.7 \\
0 \\
0 \\
0 \\
0\end{array}$ \\
\hline
\end{tabular}

\section{Discusión}

Los principales daños en los frutos estuvieron asociados a las larvas de C. splendana, muy por encima del resto de especies perforadoras de la castaña que han sido citadas en diferentes regiones de España: los lepidópteros Cydia fagiglandana (Zeller) y Pammene fasciana (L.) y el coleóptero C. elephas (Mansilla et al., 2000; Vargas Osuna et al., 2003; Torres-Vila et al., 2008). Esta mayor incidencia de C. splendana, junto con la menor intensidad de los daños en Málaga respecto de Huelva, se corresponden con trabajos anteriores realizados en las mismas zonas en los últimos 10 años (Romero, 2013).

Las diferencias significativas en la intensidad de daños causados por C. splen- 


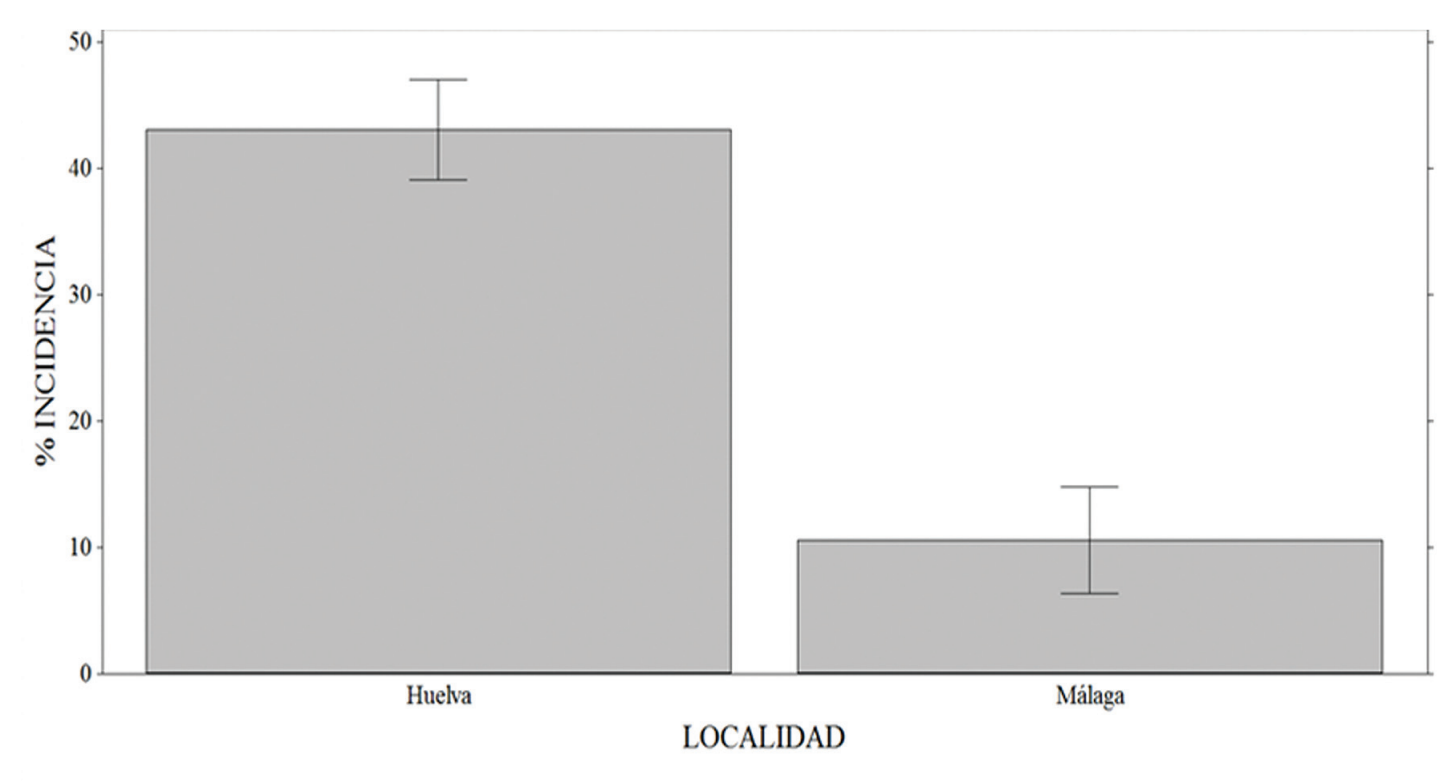

Figura 1. Valores medios ( $\pm \mathrm{SE})$ del porcentaje de daños causados por C. splendana para las zonas correspondientes a las provincias de Huelva y Málaga.

dana entre variedades puede deberse a una distinta susceptibilidad en base a características morfológicas y fenológicas de cada variedad, como se ha sugerido para explicar las diferencias encontradas en los daños causados por C. elephas en Suiza entre las variedades de castaño 'Lüina' y 'Torcion' (Sieber et al. 2007). En el caso de C. splendana, la actividad de vuelo coincide con la fase de maduración de los frutos (Mansilla et al., 2000) y la hembra selecciona el lugar de puesta en respuesta, primeramente, a estímulos olfativos (kairomonas); por ello, diferencias fenológicas entre las variedades así como en los compuestos volátiles emitidos en los estados de floración y fructificación pueden influir en el comportamiento ovipositor de la hembra. Así, por ejemplo, en ensayos de comportamiento de la especie congenérica Cydia nigricans, el contenido de volátiles durante la floración se ha relacionado con la planta huésped elegida por la hembra para realizar la puesta (Thöming et al., 2014).

\section{Conclusiones}

Nuestro estudio ha establecido las bases para la caracterización de la susceptibilidad a $C$. splendana de las variedades de castaños en Andalucía, encontrando diferencias entre variedades en cada zona productora. No obstante, en estas diferencias pueden estar implicados otros factores no relacionados con la característica varietal, como condiciones climáticas locales de los árboles o manejo de la finca (laboreo y aprovechamiento ganadero) que pueden afectar al nivel poblacional de la especie fi- 


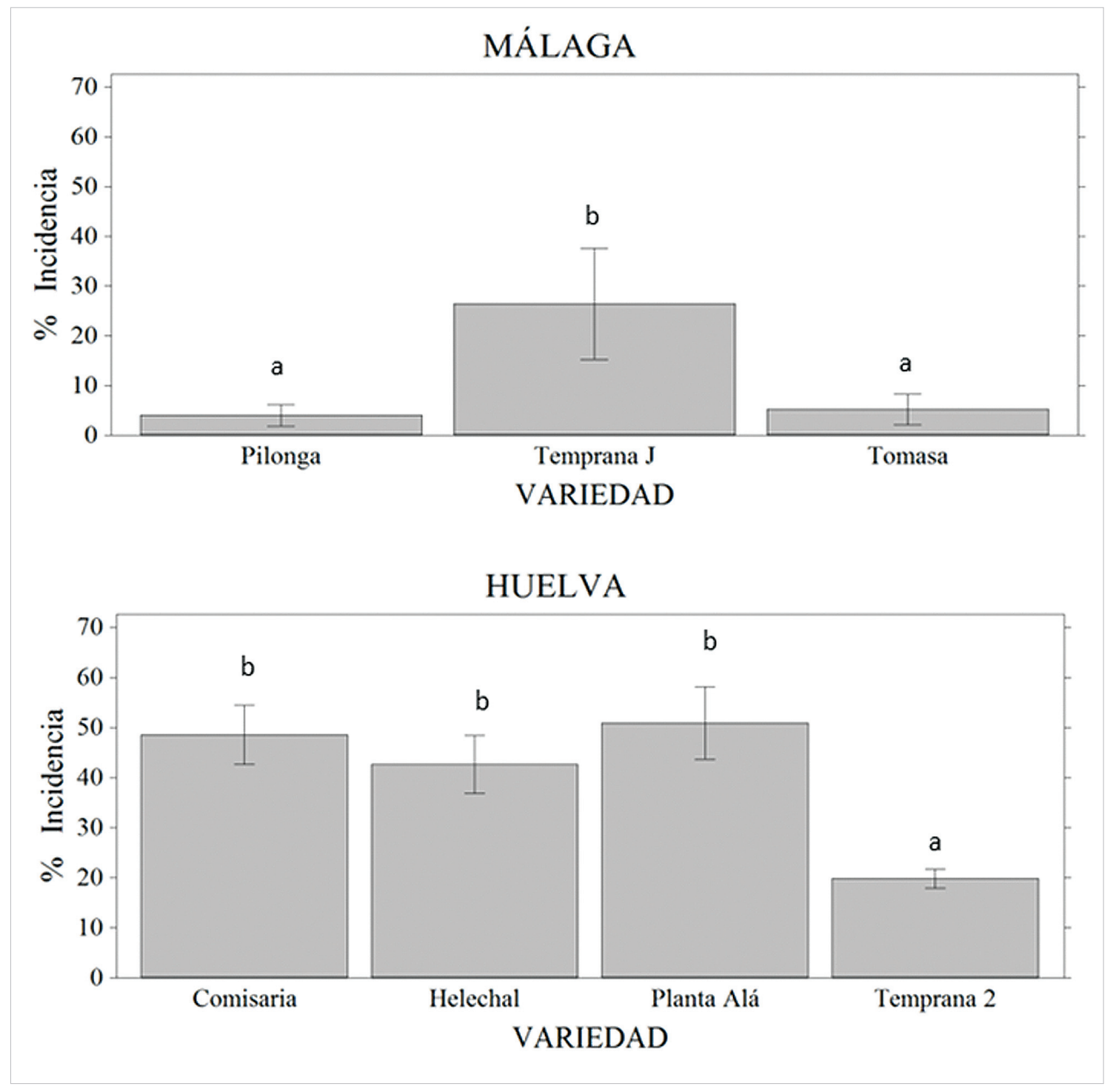

Figura 2. Valores medios ( \pm SE) del porcentaje de daños causados por $C$. splendana para diferentes variedades de las provincias de Huelva y Málaga. Barras coronadas con la misma letra, en cada zona, indica que no difieren significativamente entre sí de acuerdo con el test de comparación LSD $(p \leq 0.05)$.

tófaga. Para reducir la influencia de estos factores ambientales no controlados es necesario continuar estos estudios en años sucesivos e incorporando nuevos árboles que incrementen la precisión de los resultados.

\section{Agradecimientos}

Este estudio ha sido financiado por el Ministerio de Economía y Competitividad en el marco del Proyecto de Investigación del Plan Nacional de I+D+i AGL201348017-C2-1-R. M.A. Martín agradece la financiación a la Secretaría General de 
Ciencia y Tecnología de la Consejería de Economía, Competitividad e Innovación de la Junta de Extremadura (España).

\section{Bibliografía}

Buck, E., Russell, K., Hadonou, M. ,James, C., Blakesley, D. 2003. Isolation and characterization of polymorphic microsatellites in European chestnut (Castanea sativa Mill.). Mol. Ecol. Notes 3, 239-241.

Cobos Suárez, P. 1989. Fitopatología del castaño (Castanea sativa Miller). Bol. Sanidad Vegetal 16, $129 \mathrm{pp}$.

Fady, B. 2012. Biogeography of neutral genes and recent evolutionary history of pines in the Mediterranean Basin. Ann. For. Sci. 69, 421-428.

Gobbin, D., Hohl, L., Conza, L., Jermini, M., Gessler, C., Conedera, M. 2007. Microsatellite-based characterization of the Castanea sativa cultivar heritage of southern Switzerland. Genome 50, 1089-1103.

Lusini, I., Velichkov, I., Pollegioni, P., Chiocchini, F., Hinkov, G., Zlatanov, T., Mattioni, C. 2014. Estimating the genetic diversity and spatial structure of Bulgarian Castanea sativa populations by SSRs: implications for conservation. Conserv. Genet. 15, 283-293.

Mansilla Vázquez, J.P., Pérez Otero, R., Pintos Varela, C., Salinero Corral, C., Iglesias Vázquez, C. 2000. Plagas y enfermedades del castaño en Galicia. Xunta de Galicia. Consellería de Agricultura, Ganadería y Política Agroalimentaria. Pontevedra.

Marinoni, D., Akkak, A., Bounous, G., Edwards, K., Botta, R. 2003. Development and characterization of microsatellite markers in Castanea sativa (Mill.). Mol. Breeding 11, 127 136.

Martín, M.A, Álvarez, J.B., Mattioni, C., Cherubini, M., Villani, F., Martín, L.M. 2009. Identification and characterisation of traditional chestnut varieties of southern Spain using morphological and simple sequence repeat (SSRs) markers. Ann. Appl. Biol. 154, 389398.

Martín, M.A., Mattioni, C., Cherubini, M., Taurchini, D., Villani, F. 2010. Genetic diversity in European chestnut populations by means of genomic and genic microsatellite markers. Tree Genet. Genomes 6, 735-744.

Mattioni, C., Martín, M.A., Pollegioni, P., Cherubini, M., Villani, F. 2013. Microsatellite markers reveal a strong geographical structure in European populations of Castanea sativa (Fagaceae): evidence for multiple glacial refugia. Am. J. Bot. 100, 951-961.

Murray, M.G., Thompson, W.F. 1980. Rapid isolation of high molecular weight plant DNA. Nucleic Acids Res. 8, 4321-4326.

Pereira-Lorenzo, S., Costa R., Ramos-Cabrer A., Ribeiro C., da Silva M., Manzano G., Barreneche, T. 2010. Variation in grafted european chestnut and hybrids microsatellite reveals two main origins in the Iberian Peninsula. Tree Genet. Genom. 5, 701-715.

Romero, A. 2013. El control de los insectos carpófagos del castaño (Castanea sativa) en Andalucía mediante captura masiva con feromona sexual y evaluación de la actividad insecticida de hongos entomopatógenos. Tesis Doctoral. Universidad de Córdoba.

Schlötterer, C. 2004. The evolution of molecular markers-just a matter of fashion? Nat. Rev. Genet. 5, 63-69. 
Sieber, T.N., Jermini, M., Conedera, M. 2007. Effects of the harvest method on the infestation of chestnuts (Castanea sativa) by insects and moulds. J. Phytopathol. 155, 497-504.

Steinkellner, H., Lexer, C., Turetschek, E., Glössl, J. 1997. Conservation of (GA) n microsatellite loci between Quercus species. Mol. Ecol. 6, 1189-1194.

Thöming, G., Norl, H.R., Saucke, H., Knudsen, G.K. 2014. Pea plant volatiles guide host location behaviour in the pea moth. Arthropod Plant Interact. 8, 109-122.

Torres-Vila, L.M., Cruces Caldera, E., Sánchez González, A., Ferrero García, J.J., Ponce Escudero, F., Martín Vertedor, D., Aza Barrero, C., Rodríguez Corbacho, F., Barrena Galán, F. 2008. Dinámica poblacional y daños de Curculio elephas Gyllenhal (Col.: Curculionidae), Cydia fagiglandana Zeller, Cydia triangulella Goeze y Pammene fasciana L. (Lep.: Tortricidae) sobre Quercus y Castanea en Extremadura. Bol. San. Veg. Plagas 34, 329-341.

Vahdati, K., Pourtaklu, S.M., Karimi, R., Barzehkar, R., Amiri, R., Mozaffari, M., Woeste, K. 2015. Genetic diversity and gene flow of some Persian walnut populations in southeast of Iran revealed by SSR markers. Plant Syst. Evol. 301, 691-699.

Vargas Osuna, E., Aldebis, H.K., Alanís, R., Álvarez, J., García Castro, M. 2003. Las plagas de los castañares en Andalucía. In: El castaño en Andalucía. Consejería de Medio Ambiente. Junta de Andalucía. pp. 139-143.

Varshney, R.K., Graner, A., Sorrells, M.E. 2005. Genic microsatellite markers in plants: features and applications. Trends Biotechnol. 23, 48-55. 
